\title{
Lithium diisopropylamide (LDA) reduction of nitroarenes
}

\author{
Anlai Wang and Ed Biehl* \\ Chemistry Department, Southern Methodist University, Dallas, TX 75275 USA \\ E-mail:ebiehl@mail.smu.edu
}

\begin{abstract}
Lithium diisopropylamide (LDA) was found to reduce a wide variety of nitroarenes to the corresponding azoxyarene and aminoarene at $-40{ }^{\circ} \mathrm{C}$.
\end{abstract}

Keywords: Reduction, azoxyarenes, low-temperature synthesis

\section{Introduction}

Although LDA is commonly thought in terms of its basic properties, it has been shown to behave as a single-electron transfer (SET) donor in reactions with geminal diiodides ${ }^{1,}$ heterocycles, ${ }^{2} \alpha$ bromo imines, ${ }^{3}$ and conjugated acetylenes. ${ }^{4}$ LDA is also known to reduce certain haloarenes by donating an $\alpha$-hydrogen to the aryne intermediate in these reactions. ${ }^{5} \mathrm{We}^{6}$ recently reported the first example of LDA functioning as SET agent in its reactions with nitroarenes. In these reactions, 2-,3 and 4-chloronitrobenzene did not react with LDA at - $40{ }^{0} \mathrm{C}$ to give expected aryne products, but rather were reduced by LDA to 2,2'- 3,'3- and 4,4'-dichloroazoxybenzenes, respectively. No attempts were made to determine if chloroanilines were also formed in these reactions. Prior to this study, no low temperature reduction of nitroarenes to azoxyarenes, some of which are important dyes or exhibit significant biological activity, had been reported. Given the uniqueness and low-temperature reaction conditions of the LDA reduction, we have extended its scope a wide variety of nitroarenes and report the results herein.

\section{Results and Discussion}

Thirteen nitroarenes 1a-m were first treated with varying amounts of LDA at $-40{ }^{\circ} \mathrm{C}$ for $1 \mathrm{~h}$ and found to give mixtures of the corresponding aniline 2a-n and azoxyarene 3a-m. The best yields of the azoxyarenes were obtained by using 5 equivalents of LDA per one equivalent of nitroarene The yields are listed in Table 1 . As shown, nitropyrene $\mathbf{1 j}$, 4-chloronitrobenzene $\mathbf{1 d}$, 2-methyl-4-nitrobenzoic acid $\mathbf{1} \mathbf{j}$, and 4-nitrotoluene $\mathbf{1 e}$ gave the corresponding azoxyarenes as 
predominant products, i.e. $3 \mathbf{j}$ (83\%), 3d (53\%), 3i (37\%), and 3e (38\%), respectively. On the other hand, nitrobenzene 1a, 2-nitrobiphenyl 1h, 3,4-dimethylnitrobenzene $\mathbf{1 k}$ and 2,4dimethylnitrobenzene $\mathbf{1 n}$ gave anilines as predominant products, i.e. $\mathbf{2 a}(51 \%), \mathbf{2 h}(50 \%), \mathbf{2 k}$ (43\%), and $2 \mathbf{l}$ ( $31 \%$ ), respectively. Separation of the azoxyarenes $\mathbf{3}$ from anilines $\mathbf{2}$ was easily accomplished by simple acid-base extraction methodology. Interestingly, yields of $\mathbf{2}$ and $\mathbf{3}$ did not vary when the reduction was carried out at $-70{ }^{\circ} \mathrm{C}$, although the reactions were much slower, requiring stirring overnight for completion. Attempts to reduce 2- and 4-nitropyridine using LDA failed.

In most cases, products $\mathbf{2}$ and $\mathbf{3}$ were known compounds and thus identified by comparison to their physical properties and/or spectral data to those of known compounds. In the case of new compounds, structures were identified on the basis of spectral properties and elemental analysis.

In conclusion, the major finding of this study is that LDA reduces nitrobenzenes to azoxybenzenes and anilines at low temperatures. Prior to our work, LDA had never been implicated as a reducing agent for nitro compounds. This finding is significant in its own right. However, since the reduction occurs at low temperatures to azobenzenes as major products, it might also serve as a valuable alternative to previously reported methods all of which requires room or elevated temperatures and some require several steps. For example, one method requires a two step process at $45{ }^{0} \mathrm{C}$ which involves the conversion of nitrobenzenes to hydroxylamines followed by reduction with $\mathrm{I}_{2}$ or $\mathrm{FeCl}_{3}{ }^{7}$ Catalytic reduction using $\mathrm{NaBH}_{4}{ }^{8}$ or $\mathrm{NaBH}_{4} / \mathrm{LiCl}$ in diglyme at $125-162{ }^{\circ} \mathrm{C}^{9}$, as well as reductions in basic alcoholic refluxing media, are also

commonly used. ${ }^{10-12}$ Recently a $\mathrm{BiCl}_{3}-\mathrm{Zn}$ promoted reduction has been performed at room temperature. ${ }^{13}$

\section{Experimental Section}

General Procedures. Melting points were taken on a Mel-Temp capillary apparatus and are uncorrected with respect to stem correction. IR spectra were recorded on a Nicolet Magna-IR ${ }^{\mathrm{TM}}$ 550 FTIR instrument. ${ }^{1} \mathrm{H}$ and ${ }^{13} \mathrm{C}$ NMR spectra were recorded on a $400 \mathrm{MHz}$ Bruker AVANCE DRX-400 Multi-nuclear NMR spectrometer; chemical shifts were referenced to TMS as internal standard and coupling constants $J$ are given in $\mathrm{Hz}$.

Materials. These were obtained from commercial sources. The LDA used was obtained as a 2.0M solution in hexanes.

\section{General procedure for LDA reduction of nitroarenes 1a-m}

A $12.5 \mathrm{ml}$ portion of $2.0 \mathrm{M}$ solution of LDA ( $25 \mathrm{mmol}$ ) was added to a flame-dried flask flushed with nitrogen and containing $10 \mathrm{ml}$ of THF and the resulting mixture was cooled to $-40{ }^{\circ} \mathrm{C}$. The appropriate nitroarene $(5 \mathrm{mmol}$ ) was then added, and the resulting solution was first stirred for 5 min then allowed to warm to rt where it was quenched with sat. aq. $\mathrm{NH}_{4} \mathrm{Cl}(30 \mathrm{~mL})$. The 
resulting crude mixture was dissolved in methylene chloride and extracted with $3 \times 10 \mathrm{~mL}$ of dil $\mathrm{HCl}$. The methylene chloride extracts was dried, and evaporated to give impure $\mathbf{3}$ which was purified by flash column chromatography (silica gel) using a mixture of hexane/ethyl acetate (9:). Pertinent physical and spectral properties of the azoxybenzenes 4-6 are given below.

Table 1

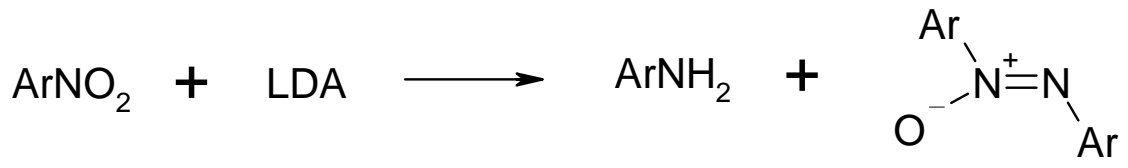

\begin{tabular}{|c|c|c|c|c|c|}
\hline Nitroarene & $\mathrm{Ar}$ & Aminoarene & Yield, \% & Azoxyarene & Yield, \% \\
\hline $1 a$ & Ph- & $2 a$ & 51 & $3 a$ & 32 \\
\hline $1 b$ & $2-\mathrm{Cl}-\mathrm{C}_{6} \mathrm{H}_{4}$ & $2 \mathbf{b}$ & 21 & $3 \mathbf{b}$ & 23 \\
\hline $1 c$ & $3-\mathrm{Cl}-\mathrm{C}_{6} \mathrm{H}_{4}$ & $2 c$ & 18 & $3 c$ & 29 \\
\hline $1 d$ & $4-\mathrm{Cl}-\mathrm{C}_{6} \mathrm{H}_{4}$ & $2 d$ & 12 & $3 d$ & 53 \\
\hline $1 e$ & $4-\mathrm{Me}-\mathrm{C}_{6} \mathrm{H}_{4}$ & $2 \mathbf{e}$ & 23 & $3 \mathbf{e}$ & 38 \\
\hline 1f & $4-\mathrm{MeO}-\mathrm{C}_{6} \mathrm{H}_{4}$ & $2 f$ & 29 & $3 f$ & 35 \\
\hline $1 \mathrm{~g}$ & 4- $\mathrm{Ph}-\mathrm{C}_{6} \mathrm{H}_{4}$ & $2 g$ & 42 & $3 g$ & 45 \\
\hline $1 h$ & 2-Ph- $\mathrm{C}_{6} \mathrm{H}_{4}$ & $2 \mathbf{h}$ & 50 & $3 h$ & 4 \\
\hline $1 \mathbf{i}$ & & $2 \mathbf{i}$ & 13 & $3 \mathbf{i}$ & 37 \\
\hline $\mathbf{1 j}$ & & $2 \mathbf{j}$ & 5 & $3 \mathbf{j}$ & 83 \\
\hline $1 k$ & 2,3-diMe- $\mathrm{C}_{6} \mathrm{H}_{3}$ & $2 \mathbf{k}$ & 43 & $3 \mathbf{k}$ & 28 \\
\hline 11 & 2,4-diMe- $\mathrm{C}_{6} \mathrm{H}_{3}$ & 21 & 31 & 31 & 24 \\
\hline $1 \mathrm{~m}$ & 1-naphthyl & $2 \mathrm{~m}$ & 10 & $3 m$ & 7 \\
\hline
\end{tabular}


Azoxybenzene (3a). $\mathrm{mp} 35-36{ }^{\circ} \mathrm{C}\left(\right.$ lit. $\left.^{14} 36{ }^{\circ} \mathrm{C}\right)$.

Bis-2-chloroazoxybenzene (3b). Red solid, mp 114-115 ${ }^{\mathrm{O}} \mathrm{C}$ (lit. ${ }^{6}$ 58-59 ${ }^{0} \mathrm{C}$. IR (KBr) $\vee 1619$ $(\mathrm{N}=\mathrm{N}), 776$ and $730 \mathrm{~cm}^{-1}$ (o-substitution); ${ }^{1} \mathrm{H}$ NMR ( $\mathrm{d}_{6}$-acetone) $\delta 6.99-7.01(1 \mathrm{H}, \mathrm{m}), 7.21-7.22$ $(1 \mathrm{H}, \mathrm{m}), 7.44-7.46(1 \mathrm{H}, \mathrm{m}), 7.58-7.62(3 \mathrm{H}, \mathrm{m}), 8.22(1 \mathrm{H}, \mathrm{d}, J=8.2 \mathrm{~Hz}) .{ }^{13} \mathrm{C}$ NMR ( $\mathrm{d}_{6}$-acetone) $\delta 116.3,118.4,124.5,125.9,126.1,126.8,127.6,128.9,130.7,125.6 .136 .3,141.6$. (Found: C, 54.1; $\mathrm{H}, 3.2 ; 10.5 . \mathrm{C}_{12} \mathrm{H}_{8} \mathrm{~N}_{2} \mathrm{OCl}_{2}$ requires $\mathrm{C}$, 54.0; $\left.\mathrm{H}, 3.0 ; \mathrm{N}, 10.5\right)$.

Bis-3-chloroazoxybenzene (3c). Light yellow solid, mp 105-106 ${ }^{\circ} \mathrm{C}$ (lit. ${ }^{6} 96-97{ }^{\circ} \mathrm{C}$ ).

Bis-4-chloroazoxybenzene (3d). Light yellow solid, mp $158-159{ }^{\circ} \mathrm{C}\left(\right.$ lit. $\left.{ }^{6} 158{ }^{\circ} \mathrm{C}\right)$.

Bis-4-methylazoxybenzene (3e) $\mathrm{mp} 63-65^{\circ} \mathrm{C}\left(\right.$ lit $^{14} 66-68{ }^{\circ} \mathrm{C}$ )

Bis-4-methoxyazoxybenzene (3f) $\mathrm{mp} 115-116{ }^{\circ} \mathrm{C}$ (lit. ${ }^{15} 116-118{ }^{\circ} \mathrm{C}$ )

Bis-4-phenylazoxybenzene (3g). Yellow solid, mp 212-214 ${ }^{\circ} \mathrm{C}$. IR (KBr) v: 3056, 3033, 1598, 1481, 1461, 1443, 843, $762 \mathrm{~cm}^{-1} .{ }^{1} \mathrm{H}$ NMR $\left(\mathrm{CDCl}_{3}\right) \delta 7.41(\mathrm{~m}, 2 \mathrm{H}), 7.51(\mathrm{~m}, 4 \mathrm{H}), 7.68(\mathrm{t}, J=$ $8.8 \mathrm{~Hz}, 1 \mathrm{H}$ ), 7.75 (d,d, $\left.J_{1}=8.8 \mathrm{~Hz}, J_{2}=7.6 \mathrm{~Hz}, 4 \mathrm{H}\right), 8.35$ (d, $\left.J=8.8 \mathrm{~Hz}, 2 \mathrm{H}\right), 8.43$ (d, $J=8.8$ $\mathrm{Hz}, 2 \mathrm{H}) .{ }^{13} \mathrm{C}$ NMR $\left(\mathrm{CDCl}_{3}\right) \delta 122.9,126.3,127.2,127.4,127.5,127.9,128.3,129.0,129.1$, 139.6, 140.2, 142.4, 143.3, 144.6, 147.5. Calcd for $\mathrm{C}_{24} \mathrm{H}_{18} \mathrm{~N}_{2} \mathrm{O}$ : C, 82.3; H, 5.2; N, 8.0. Found: C. 82.1; H, 5.2; N, 7.9.

4-Carboxy-3-chloroazoxybenzene (3i). Brown solid, mp $290^{\circ} \mathrm{C}$ (decom). IR $\left(\mathrm{CDCl}_{3}\right) \vee 3410$ (br), 2975, 1707, 1600, 1465, 1249, 1045, 831, 757. ${ }^{\mathrm{I}} \mathrm{H}$ NMR (DMSO-d 6 ) $\delta 7.72$ (d, $J=8.60,1$ H), 7.79 (d, $J=8.8 \mathrm{~Hz}, 1 \mathrm{H}), 8.21$ (d, $J=8.8 \mathrm{~Hz}, 1 \mathrm{H}$ ), 8.34 (d, $J=8.6 \mathrm{~Hz}, 1 \mathrm{H}), 8.52(\mathrm{~s}, 1 \mathrm{H})$, 8.57 (s, $1 \mathrm{H}$ ), Anal. Calcd for C. Calcd. For $\mathrm{C}_{14} \mathrm{H}_{7} \mathrm{~N}_{2} \mathrm{O}_{5} \mathrm{Cl}_{2}:$ C, 47.5; H, 2.0; N, 7.9. Found: C, 47.6; H, 1.9; N, 7.8.-

Azoxy-1-pyrene (3j). Yellow solid, mp 240-242 ${ }^{\circ} \mathrm{C}$. IR (KBr) v: 3037, 1594, 1499, 1154, 1022, 838, $783 \mathrm{~cm}^{-1} .{ }^{1} \mathrm{H}$ NMR $\left(\mathrm{CDCl}_{3} \delta 8.09(7.6, \mathrm{~Hz} 1 \mathrm{H}), 8.18(\mathrm{~m}, 5 \mathrm{H}), 8.27-8.37(\mathrm{~m}, 8 \mathrm{H}), 8.67(\mathrm{~d}\right.$, $J=8.8 \mathrm{~Hz}, 1 \mathrm{H}$ ), 8.78 (d, $J=8.8 \mathrm{~Hz}, 1 \mathrm{H}), 8.91$ (d, $J=8.8 \mathrm{~Hz}, 1 \mathrm{H}), 9.78$ (d, $J=8.8 \mathrm{~Hz}, 1$ H). Anal. Calcd for C. Calcd for $\mathrm{C}_{32} \mathrm{H}_{18} \mathrm{~N}_{2} \mathrm{O}$; C, 86.1; H, 4.1:, N, 6.3. Found: C, 86.2; H, 4.2; N, 6.4.

Bis-(3,4-dimethyl)azoxybenzene (3k). Yellow solid, mp 141.-142 ${ }^{\circ} \mathrm{C}$. IR $\left(\mathrm{CDCl}_{3}\right) \vee 3018,2969$, 2918, 1609, 1491, 1465, 1317, 867, 821. ${ }^{1} \mathrm{H}$ NMR $\left(\mathrm{CDCl}_{3}\right) \delta 2.34$ (s, $\left.3 \mathrm{H}\right), 2.35$ (s, $\left.3 \mathrm{H}\right), 2.36$ (s, $3 \mathrm{H}), 2.38$ (s, $3 \mathrm{H}), 7.24$ (d, $J=8.4 \mathrm{~Hz}, 2 \mathrm{H}), 8.03(\mathrm{~m}, 3 \mathrm{H}), 8.09(\mathrm{~s}, 1 \mathrm{H}) .{ }^{13} \mathrm{C} \mathrm{NMR}\left(\mathrm{CDCl}_{3}\right) \delta$ 19.6, 19.8, 19.9, 20.0, 119.6, 122.9, 123.0, 126.8, 129.7, 129.8, 129.7, 129.8, 136 (3l):.8, 137.2, 138.7, 140.5, 142.2, 146.4. Calcd for C. Calcd for $\mathrm{C}_{16} \mathrm{H}_{18} \mathrm{~N}_{2} \mathrm{O}$ : C, 75.6; H, 7.1; N, 11.0. Found: C. 75.7; H, 7.2; N, 10.9 .

(2,4-Dimethyl)azoxybenzene (3l). Colorless crystals mp .56-57.5 ${ }^{\circ} \mathrm{C} .{ }^{1} \mathrm{H}$ NMR $\left(\mathrm{CDCl}_{3}\right) \delta 2.35$ (s, 6 H), 2.38 (s, 3 H), 2.48 (s, 3 H), 7.08 (d, $J=8.4$ Hz, 2 H), 7.12 (s, 2 H), 7.56 (d, $J=8.8$ Hz, 1 $\mathrm{H}), 8.12(\mathrm{~d}, J=8.4 \mathrm{~Hz}, 1 \mathrm{H}) .{ }^{13} \mathrm{C}$ NMR $\left(\mathrm{CDCl}_{3}\right) \delta 18.9,19.0,21.5,21.7,122.0,124.0,127.0$, 127.4, 131.4, 131.8, 132.7, 135.1, 129.2, 140.5, 140.9, 147.9. Anal. Calcd for $\mathrm{C}_{16} \mathrm{H}_{18} \mathrm{~N}_{2} \mathrm{O}$ : C, 75.6; H, 7.1; N, 11.0. Found: C. 75.7; H, 7.2; N, 10.9. 


\section{Acknowledgements}

This work was sponsored, in part, by grants from the Welch Foundation, Houston, TX and the Petroleum Research Fund, administered by the American Chemical Society. The high resolution mass spectral analyses were performed by the Washington University Mass Spectrometry Resource, an NIH Research Resource (Grant No. P41RR0954).

\section{References}

1. Ashby, E. C.; Deshpande A. K.;. Patil, G. S. J. Org. Chem. 1995, 60, 663.

2. Newkome G. R.; Hager D. C. J. Org. Chem. 1982, 47, 599.

3. Kimpe, N. D.; Yao, Z. P.; Schamp, N. Tetrahedron Lett. 1986, 27, 1707.

4. Shen C.; Ainsworth, C. Tetrahedron Lett. 1979, $20,89$.

5. Wittig, G.; Rentzea, C. N.; Rentzea, M. Liebigs Ann. 1971, 744.

6. Tandel S.; Wang, A.; Holdeman, T. C.; Zhang H.; Biehl E. R. Tetrahedron 1998, 54, 15147.

7. . Borah, H.; Prajapati D.; Sandhy, J.; Ghosh A. C. Tetrahedron Lett. 1994, 35, 3167.

8. Rondestvedt, C. S.; Johnson T. A. Synthesis 1977, 12, 850.

9. Yang, C.; Pittman, C. U. Synth. Commun. 1998, 28, 2027.

10. Ogata, Y.; Mibae, J. J. Org. Chem. 1962, 27, 2048.

11. Prato, M.; Quintily, U.; Scapol, L.; Scorrano. G. Bull. Soc. Chim. Fr. 1987, 99.

12. Prato, M.; Quintlily, U.; Scorrano, G. Gazz.Chim. Ital. 1988, 118, 421.

13. Ren, P.; Pan, S.; Dong, T.; Wu, S. Synth. Commun. 1996, 26, 3903

14. Gore P. H.; Wheeler, O. H. J. Am. Chem. Soc. 1956, 78, 2160.

15. Porter R. S.; Johnson, J. E. J. Phys. Chem. 1962, 66, 1826. 